\title{
Comprehensive dissection of transcript and metabolite shifts during seed germination and post-germination stages in poplar
}

Chunpu Qu $u^{1,2}$, Zhuang Zuo ${ }^{1,2}$, Lina Cao ${ }^{1,2}$, Jiahuan Huang ${ }^{3}$, Xue Sun ${ }^{1,2}$, Peng Zhang ${ }^{2}$, Chengjun Yang ${ }^{2}$, Lixin Li ${ }^{4}$, Zhiru Xu ${ }^{1,3^{*}}$ and Guanjun Liu ${ }^{1,2^{*}}$

\begin{abstract}
Background: Seed germination, a complex, physiological-morphogenetic process, is a critical stage in the life cycle of plants. Biological changes in germinating seeds have not been investigated in poplar, a model woody plant.

Results: In this study, we exploited next-generation sequencing and metabolomics analysis and uncovered a series of significantly different genes and metabolites at various stages of seed germination and post germination. The $K$ means method was used to identify multiple transcription factors, including AP2/EREBP, DOF, and YABBY, involved in specific seed germination and post-germination stages. A weighted gene coexpression network analysis revealed that cell wall, amino acid metabolism, and transport-related pathways were significantly enriched during stages 3 and 5, with no significant enrichment observed in primary metabolic processes such as glycolysis and the tricarboxylic acid cycle. A metabolomics analysis detected significant changes in intermediate metabolites in these primary metabolic processes, while a targeted correlation network analysis identified the gene family members most relevant to these changing metabolites.

Conclusions: Taken together, our results provide important insights into the molecular networks underlying poplar seed germination and post-germination processes. The targeted correlation network analysis approach developed in this study can be applied to search for key candidate genes in specific biochemical reactions and represents a new strategy for joint multiomics analyses.
\end{abstract}

Keywords: Poplar, Seed germination and post-germination stages, Transcriptomics, Metabolomics, Data integration, Candidate genes

\section{Background}

Plant seed germination and its immediate aftermath is a very complicated biological process involving a series of morphological, physiological, and biochemical changes under environmental influence [1-5]. Extensive research at both physiological and morphological levels has been performed on germination and post-germination processes of herbaceous plants such as Arabidopsis [6-9], soybean [10-12], rice [13-17], and barley [18-20]. In recent years, an 'omics' approach has been used to obtain information on changes in the levels of an entire

\footnotetext{
*Correspondence: xuzhiru2003@126.com; liuguanjun2013@nefu.edu.cn ${ }^{1}$ State Key Laboratory of Tree Genetics and Breeding (Northeast Forestry University), School of Forestry, Northeast Forestry University, Harbin 150040, People's Republic of China

Full list of author information is available at the end of the article
}

range of metabolites, proteins, and gene transcripts [21-28]. The results of such studies provide an integrated view of the biological process of seed germination and post-germination events.

Poplar is a model woody plant that has long been studied, including in terms of physiological and morphological changes during germination [29]. Previous research on rapid asexual propagation and tissue culture of poplar has been highly successful [30, 31]. In comparison, few studies have been carried out on molecular mechanisms of poplar seed germination (sexual reproduction), although several preliminary reports on the relationship between seed germination and environment have recently appeared [28, 32-34]. Investigation of the molecular mechanism of poplar seed germination and its immediate

(C) The Author(s). 2019 Open Access This article is distributed under the terms of the Creative Commons Attribution 4.0 International License (http://creativecommons.org/licenses/by/4.0/), which permits unrestricted use, distribution, and 
aftermath may be key to the elucidation of this process in woody plants.

By definition, seed germination begins with the uptake of water by the mature dry seed and terminates with the protrusion of the radicle through the seed envelope. On the basis of changes in fresh weight during seed germination, a three-phase germination model has been widely adopted in previous studies. The three phases of this model are as follows: I, rapid imbibition of water, which occurs as long as all matrices and cell contents are fully hydrated; II, limited water uptake; and III, increasing water uptake accompanied by embryo axis elongation and breakthrough of covering layers to complete germination (visible germination) $[1,35,36]$. Seeds subsequently enter a post-germination stage until expansion of the first true leaf. In the present study, we used these three phases as a foundation and divided the poplar seed germination process into six stages ranging from stage 2, rapid imbibition of water, to stage 6 , expansion of the first true leaf, with seeds before imbibition defined as stage 1 . Using next-generation sequencing and highperformance liquid chromatography-mass spectrometry (HPLC-MS/MS), we generated transcriptome and metabolome data for six seed-germination stages in poplar. We then applied $K$-means clustering and weighted gene coexpression network analysis (WGCNA) to identify stage-specific gene clusters and network modules. In addition, we found that genes involved in cells and stress response were enriched at certain stages, whereas primary metabolic processes such as glycolysis and the tricarboxylic acid cycle (TCA) did not change significantly. Network analysis of enriched modules at different stages revealed a series of highly connected hub genes involved in transport, cell walls, and signaling. Finally, targeted correlation network analysis (TCNA) allowed us to find the most relevant gene family members potentially related to metabolite changes. Taken together, our results lay a foundation for further biochemical and functional analysis of poplar seed germination and its aftermath.

\section{Results}

\section{Global analysis of RNA-Seq data}

The poplar seed germination process was divided into stages 2 to 4 of the seed germination stages defined by Bewley (1997) [1]. As assessed by changes in seed fresh weights after water absorption, periods of rapid and slow water absorption were defined as stages 2 and 3, respectively (Fig. 1a and Additional file 1: Table S1), while the hypocotyl extension period was defined as stage 4 . We collected samples at a total of six stages: stages 2 to 4 , the dry seed phase (stage 1), and post-germination stages of cotyledon unfolding (stage 5) and true-leaf unfolding (stage 6). A schematic representation of the experimental setup is shown in Fig. 1b.

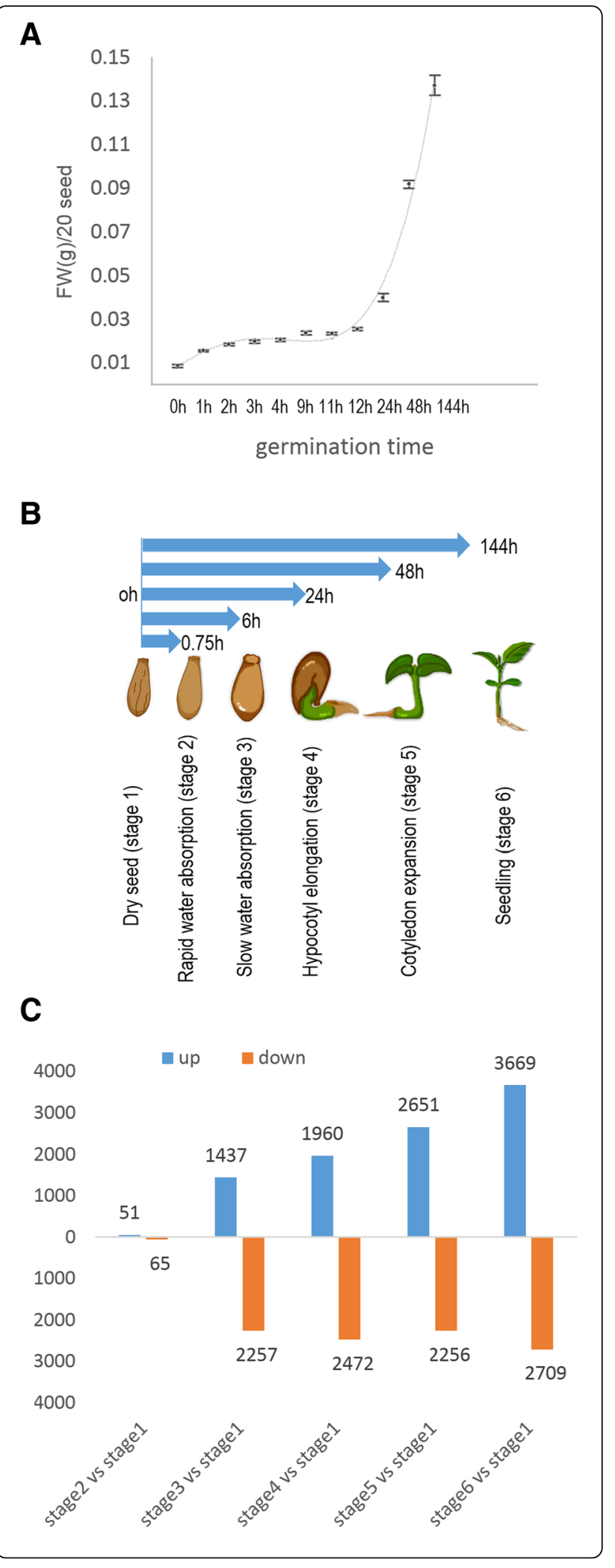


(See figure on previous page.)

Fig. 1 Schematic representation of poplar seed germination and the number of significantly changed transcripts between successive stages during poplar germination and post germination. a Changes in fresh weight after seed imbibition. $\mathbf{b}$ Schematic representation of the experimental setup. c Summary of the number of significantly changed transcripts between successive stages during poplar germination and post germination

For samples from each stage, the average number of raw reads per library was approximately 27 million (Additional file 2: Table S2), with $72 \%$ of these raw reads mapped to the poplar reference genome. Normalized read counts were calculated as FPKM values (expected number of fragments per kilobase of transcript sequence per millions of base pairs sequenced). A total of 63,498 genes, which corresponds to approximately $90 \%$ of the 73,000 genes predicted in the poplar genome, were found to be expressed during seed germination and post-germination stages. Based on the results of differential expression analysis using NOISeq, up- and downregulated genes were identified for each pairwise comparison according to the criteria of fold change $\geq 2$ and $p \geq 0.8$. The number of observed transcript changes was as follows: 116 between stages 1 and 2 (51 up, 65 down), 3694 between stages 1 and 3 (1437 up, 2257 down), 4432 between stages 1 and 4 (1960 up, 2472 down), 5207 between stages 1 and 5 (2651 up, 2256 down), and 6378 between stages 1 and 6 (3669 up, 2709 down). Compared with other stages, fewer changes in the number of transcripts were observed between stages 1 and 2. The number of genes with significant expression changes between successive time points during poplar germination and post germination is shown in Fig. 1c.

\section{Identification of temporal expression trends across poplar seed transcriptomes during germination and post germination}

To reveal the variation of differentially expressed genes (DEGs), K-means clustering was used to group and visualize RNA-Seq expression profiles of all genes differentially expressed during seed germination at each given time point, which corresponded to 10,148 genes in total. All 24 clusters are shown in Fig. 2a, and their gene IDs are listed in Additional file 3: Table S3. Each identified gene cluster could be assigned to a specific germination stage on the basis of its highest expression: clusters 5 , 18,19 , and 24 to stage 2; clusters 2 and 22 to stage 3; clusters $3,13,15$, and 21 to stage 4 ; clusters 4,10 , and 20 to stage 5 ; and clusters $1,6-9,11,12,14,16,17$, and 23 to stage 6 .

Clusters with similar expression trends were further combined into nine superclusters (Fig. 2a). To understand the distribution of genes in these superclusters, an enrichment analysis was performed with the MapMan tool to identify specific categories [37]. In supercluster 1, the top enriched MapMan category was signaling, followed by protein synthesis, glycolysis, and oxidative pentose phosphate pathway (OPP). The top enriched category in supercluster 2 was also protein synthesis, followed by signaling, RNA regulation of transcription, lipids, and protein degradation. Protein degradation was predominant in supercluster 3, followed by protein synthesis, signaling, major $\mathrm{CHO}$ metabolism, and DNA synthesis/chromatin structure. The top enriched categories in supercluster 4 were protein synthesis, RNA regulation of transcription, lipids, and major $\mathrm{CHO}$ metabolism; those in supercluster 5 were cell walls, RNA regulation of transcription, lipids, and development. In supercluster 6 , the most enriched category was RNA regulation of transcription, which was followed by cell walls, signaling, and transport. Superfamily 7 had the largest number of highly enriched categories, including protein synthesis, RNA regulation of transcription, amino acid metabolism, and lipids. In supercluster 8, the top enriched MapMan category was protein degradation, followed by signaling, cell walls, and transport. Most categories in superfamily 9 were only lowly enriched, with only protein degradation and transport having relatively high enrichment. Noteworthily, two categories, TCA/org transformation and mitochondrial electron transport/ATP synthesis, were always lowly enriched. These results indicate that the number of active genes associated with energy, TCA, and substance conversion was basically constant (Fig. 2b).

Genes related to RNA regulation of transcription, mostly encoding transcription factors, were relatively highly enriched. Five types of transcription factors, $C P P$ (Zn), AP2/EREBP, EIL, ARR, and DOF, were only expressed at one stage (Fig. 2c and Additional file 4: Table S4). $Y A B B Y$ transcription factors were specifically expressed during early germination stages, and EIN3-like (EIL) and ARR were only expressed at the end of germination (Fig. 2c). These results are consistent with previous research. For example, Howell et al. (2009) found that expression levels of $A P 2 / E R E B P$ family genes increased during early stages of seed germination in rice. This family is considered to be related to water uptake and $A B A$ signaling during early rice seed germination [25]. The dynamic and stage-specific expression patterns of these transcription factors probably reflect their key functions during different germination stages.

\section{Coexpression network analysis with WGCNA}

To understand the biological process of poplar seed germination and its aftermath from the perspective of the overall network, a coexpression network analysis with WGCNA was applied (Fig. 3a). All the DEGs at 


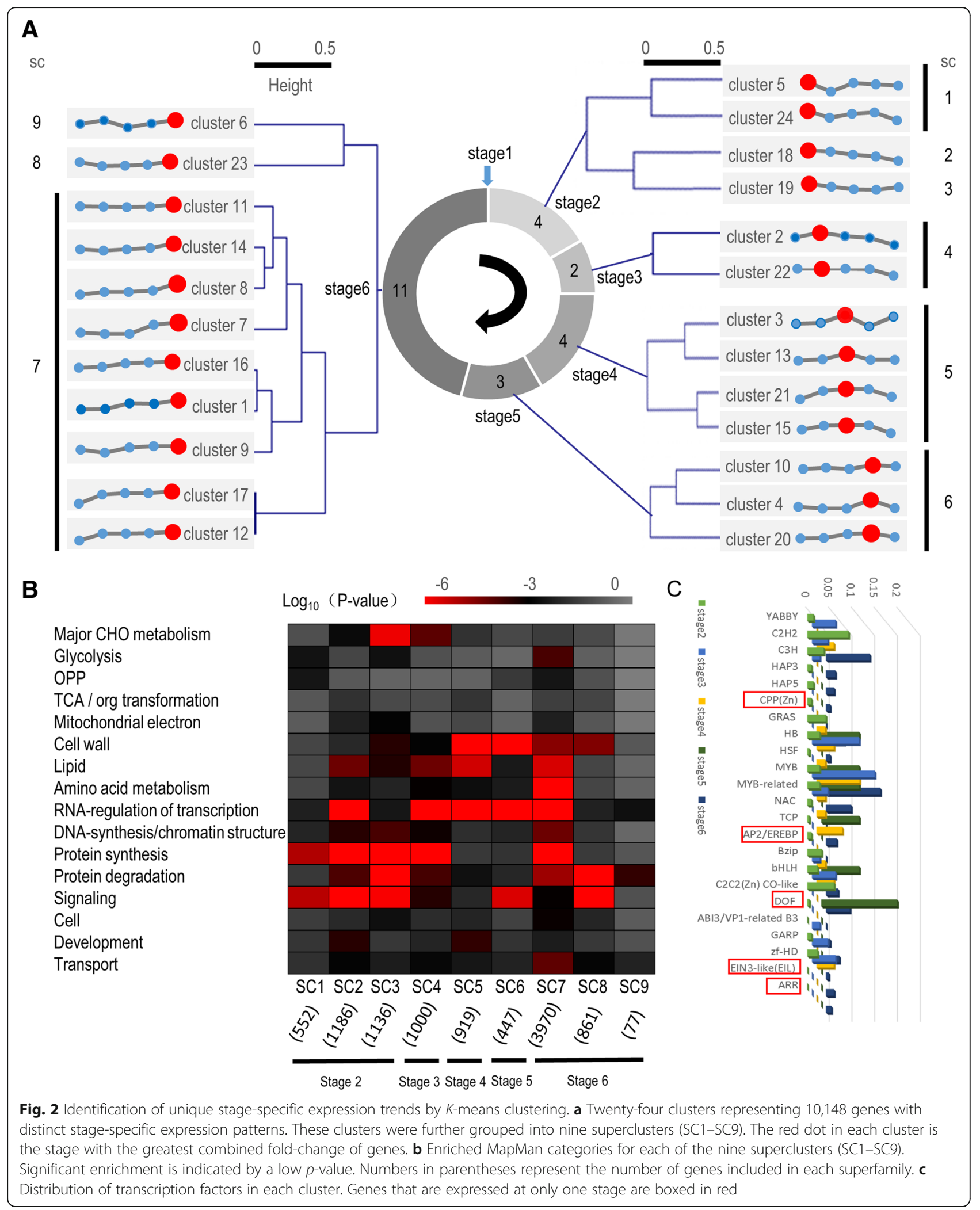




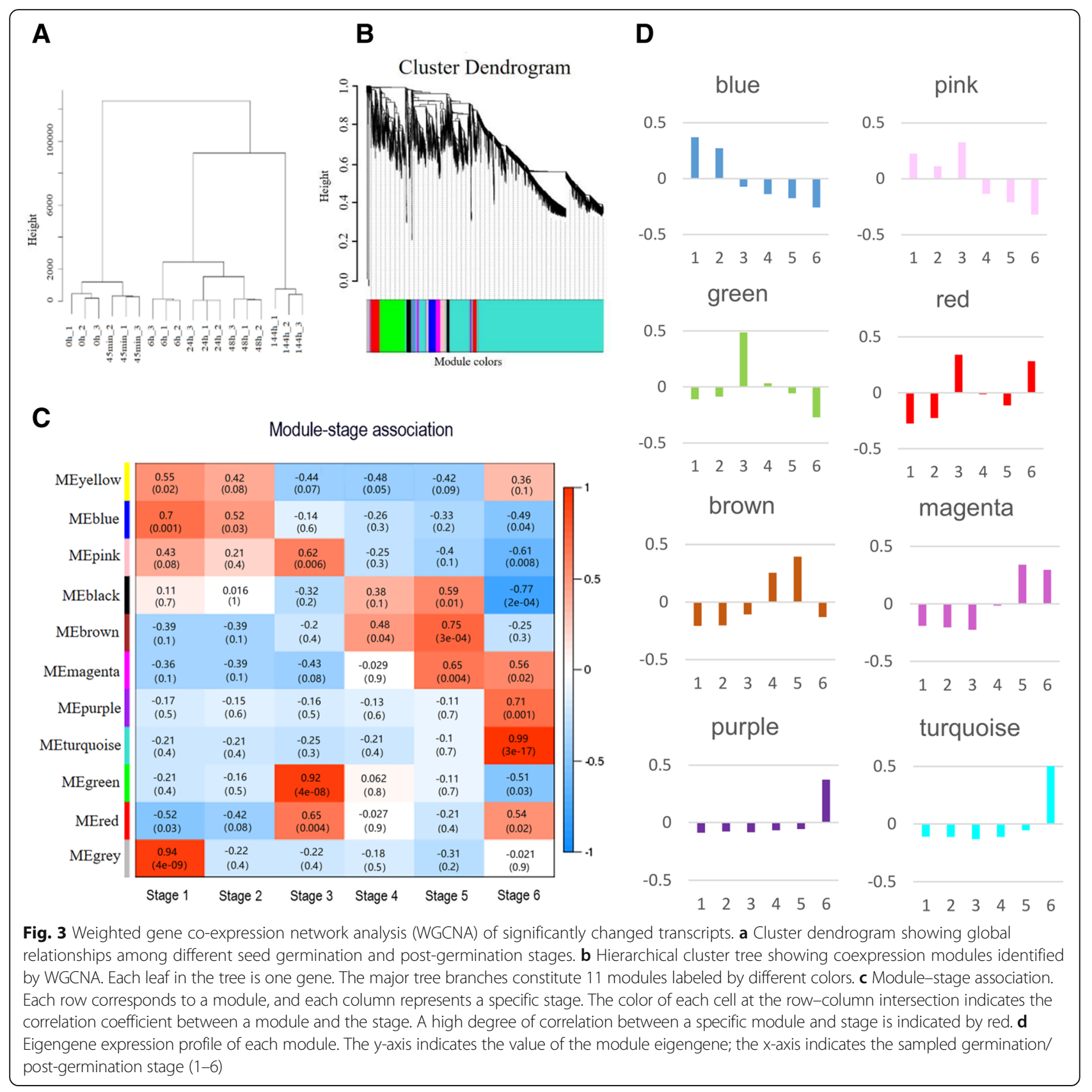

different stages were used to construct the coexpression network. Genes with similar expression patterns were classified into the same module, with colors used to distinguish among different modules (Fig. 3b). As shown in the hierarchical cluster tree in Fig. 3a, 10 different coexpression modules were identified by WGCNA. Correlation analysis demonstrated that these modules corresponded to a germination or post-germination stage-specific distribution pattern (Fig. 3c). Compared with other modules, for example, genes in the green module had the highest correlation with stage 3 ( $p=4 \times$ $\left.10^{-8}, r=0.92\right)$, whereas genes in the brown module displayed the highest correlation with stage $5(p=3 \times$ $\left.10^{-4}, r=0.75\right)$. Genes in the pink module were relatively highly correlated with stages 1,2 , and 3 (Fig. 3c).

The module eigengene (ME), the first principal component of a gene module, represents the gene expression profile of each module except for the gray module (Miscellaneous). The eigengene expression profiles of eight groups of modules are shown in Fig. 3d. The ME of the blue module had higher expression levels during stages 1 and 2. MEs of green, pink, and red modules were expressed at higher levels during stage 3, while those of brown and magenta modules had higher expression 
levels during stages 4 and 5 . The MEs of purple and turquoise modules had their highest expressions during stage 6.

To understand changes in biological processes in different modules, Fisher's exact test was applied to identify enriched pathway categories and their mobilization trends in each module. The results of this test are given in Additional file 5: Table S5. The mobilization trend of enriched categories in different colored modules was obtained by comparing the number of background genes. Genes in the yellow module were associated with significant enrichment of seven categories: major $\mathrm{CHO}$ metabolism, glycolysis, RNA regulation of transcription, protein degradation, signaling, development, and cells. The blue module was significantly correlated with stages 1 and 2, with 12 significantly enriched categories identified (Additional file 5: Table S5). The number of enriched categories varied among the other modules. The smallest number of significantly enriched pathways, one each, was found in magenta and purple modules in the categories of signaling and RNA regulation of transcription, respectively.

Among the 10 modules, the green module was most strongly associated with stage 3 , while the brown module was most relevant to stage 5 (Fig. 3c). The main analysis was thus performed on these two modules. Protein synthesis and degradation pathways were more active in the green module, whereas protein synthesis-related pathways were inhibited in the brown module. In the green module, the number of protein degradation pathway genes was elevated, but the increase was not significant (Fig. 4). The participation of protein synthesis and degradation pathways thus changed with different stages of germination. Surprisingly, the activity of the TCA cycle pathway was not significantly different among modules, consistent with the results shown in Fig. 2 and implying that the activity of this pathway is stable throughout seed germination and post germination.

WGCNA can also be used to construct gene networks in which each node represents a gene and the connecting lines between nodes, called edges, represent coexpressionrelated genes. The node gene with the highest connectivity, the hub gene, may play an important role in different modules. Brown and green module networks are shown in Fig. 5a and b, respectively. Some of the more interconnected node genes were those of transcription factors. For example, potri.015 g141800, the transcription factor gene with the highest connectivity in the green module, encodes ANL2 (ANTHOCYANINLESS 2), a homeodomain protein belonging to the HD-GLABRA2 group that is highly similar to Arabidopsis AT4G00730 sequences. In a recent study, this gene was thought to be involved in the regulation of the root development process in Arabidopsis [38, 39], and its expression has been found to be significantly reduced in somatic embryo germ-defective plants of Norway spruce [40]. ANL2 may therefore play a development-related regulatory role in poplar seed germination. The potri.016 g060600 gene, named ZFP8 (ZINC FINGER PROTEIN 8), encodes a nuclear $\mathrm{C} 2 \mathrm{H} 2$ zinc-finger protein and was found to have high connectivity within the brown module. In Arabidopsis, the protein encoded by ZFP8 is a negative regulator of gibberellin-inducible seed germination and plays a key role in germination and seedling development [41]. Some of the less interconnected transcription factors, such as a WUSCHEL-related homeobox 11 transcription factor (potri.019 g040800) found in the green module, are thought to be related to the germination of rice seeds [25]. In addition, a $Y A B B Y$ family member (potri.009 g000100) was located in the brown module; this transcription factor may play a key role in the germination of soybean seeds [42]. All of the above data are detailed in Additional file 6: Table S6.

Some highly connected functional genes were also found in different modules. The distribution statistics of high-connectivity-node functional genes in brown and green modules are given in Fig. 5c. In the green module, highly connected node genes associated with proteins, transport, signaling, and mitochondrial electron transport/ATP synthesis predominated, whereas more of such genes in the brown module were related to PS, cell walls, and lipid metabolism. One noteworthy issue is that these high-connectivity genes are not involved in key primary metabolic pathways such as glycolysis, TCA, and OPP.

To understand the types of highly connected genes in different modules, we analyzed the top 10 node genes with the highest connectivity distribution (Fig. 5d). In the green module, all high-connectivity genes belonged to protein categories, whereas functions of those in the brown module were distributed into the categories of PS, proteins, cell walls, redox, and signaling. All functional gene IDs and connectivities are listed in Additional file 7: Table S7.

\section{Primary metabolite analysis}

In this study, an untargeted metabolomics assay was performed to detect metabolic changes at different stages of seed germination and post germination. Because glycolysis, TCA, and pentose phosphate pathways play crucial roles in plant primary metabolism, changes in these three pathways during poplar seed germination were studied using metabolomics. Given the importance of the urea cycle and central amino acid metabolism, metabolites of those two pathways were also considered. A total of 19 metabolites related to the above five pathways were identified. These metabolites experienced significant changes in content during at least one stage. All metabolites and their patterns of changes are shown in Fig. 6a. Four metabolites in the glycolysis pathway were identified. Among them, glucose 1-phosphate and 


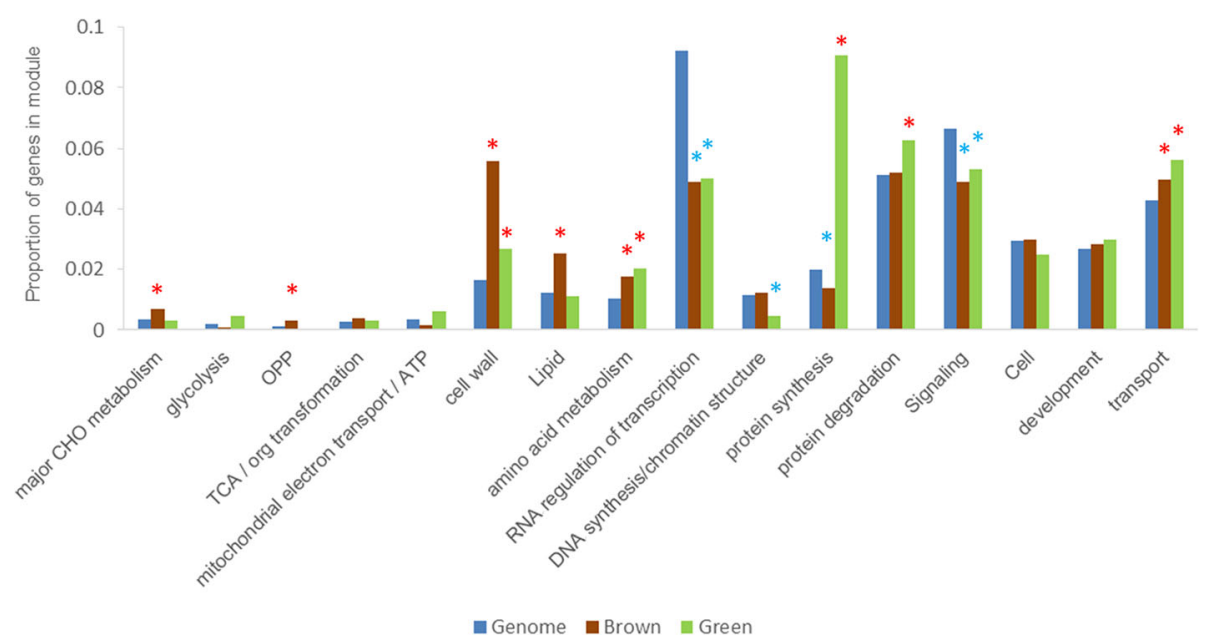

Fig. 4 Enrichment pattern of different pathway categories in brown and green modules. The frequency of transcripts in each pathway category was calculated as a percentage of the cluster and compared with the percentage of the genome. Overrepresented and underrepresented functional groups in each cluster, as determined by Fisher's exact test $(p \leq 0.05)$, are indicated with red and blue asterisks, respectively. For a complete list of all functional groups in all clusters, see Additional file 5: Table S5

glucose 6-phosphate are two main components of the hexose phosphate pool; the former is the decomposition product of starch, while the latter is closely related to glycolysis and pentose phosphate pathways. Glucose 6-phosphate was significantly increased at stage 2, while glucose 1-phosphate was significantly increased at stage 3. Both had their highest contents at stage 5 . The other two substances were dihydroxyacetone phosphate and phosphoenolpyruvate. Dihydroxyacetone phosphate was significantly increased during stages 3 to 6 , while phosphoenolpyruvate was only significantly increased at stages 5 and 6 . Noteworthily, the patterns of changes in the contents of these four metabolites were extremely similar, as their contents increased significantly during the later stages of seed germination (Fig. 6a and Additional file 8: Table S8).

Five metabolites were closely related to the TCA: citrate, cis-aconitate, alpha-ketoglutarate, succinate, and oxaloacetate. Changes in the contents of citrate and cis-aconitate followed similar trends, i.e., significant increases at stages 2,4 , and 5 , whereas levels of alpha-ketoglutarate increased during stages 1 to 5 but were only significantly increased at stage 4. Oxaloacetate underwent significant decreases at stages 2,3 , and 6 , while succinate was significantly decreased at stages 3 and 4 and significantly increased at stage 6. In regard to metabolites related to the urea cycle, argininosuccinic acid exhibited a significant increase from stages 2 to 5 , ornithine was significantly increased during stages 2 to 6 , and citrulline was only increased significantly at stage 4 . Two metabolites in the pentose phosphate pathway, ribulose 5-phosphate and ribulose 1,5-bisphosphate, exhibited a consistent pattern of change, with significant increases from stages 2 to 6 . In the central amino acid pathway, glutamate significant increased at stage 5, and glutamine significantly increased from stages 4 to 6 . Aspartate was significantly increased at stages 5 and 6 , while asparagine was significantly increased at stage 6 .

\section{Data integration analysis}

To investigate the characteristics of primary metabolic processes during seed germination, transcriptome and metabolome data for the entire germination process were subjected to an integration analysis. Figure $6 \mathrm{a}, \mathrm{b}$ and Additional file 8: Table S8 and Additional file 9: Table 59 show changes in primary metabolites and the expression patterns of related genes. We found that many genes encoding the same enzyme exhibited different expression patterns. For example, both potri.015 g131100 and potri.006 g116800 genes encode enolase but had opposite expression patterns, and the change in their target metabolite, phosphoenolpyruvate, was not consistent with either expression pattern. A similar phenomenon was observed for metabolic pathways such as the TCA cycle. These results indicate that the regulation of primary metabolism during poplar seed germination is very complicated; one possible reason is that the modulation of enzymatic activity in the primary metabolic pathways happens post-transcriptionally.

To reveal the interrelationships of genes and their associated metabolites, TCNA was applied to intrinsically link RNA-Seq and metabolomic data. Detailed results of this analysis are shown in Fig. 7 and Additional file 10: Table S10. Through this targeted correlation analysis, family members most strongly correlated to metabolites 


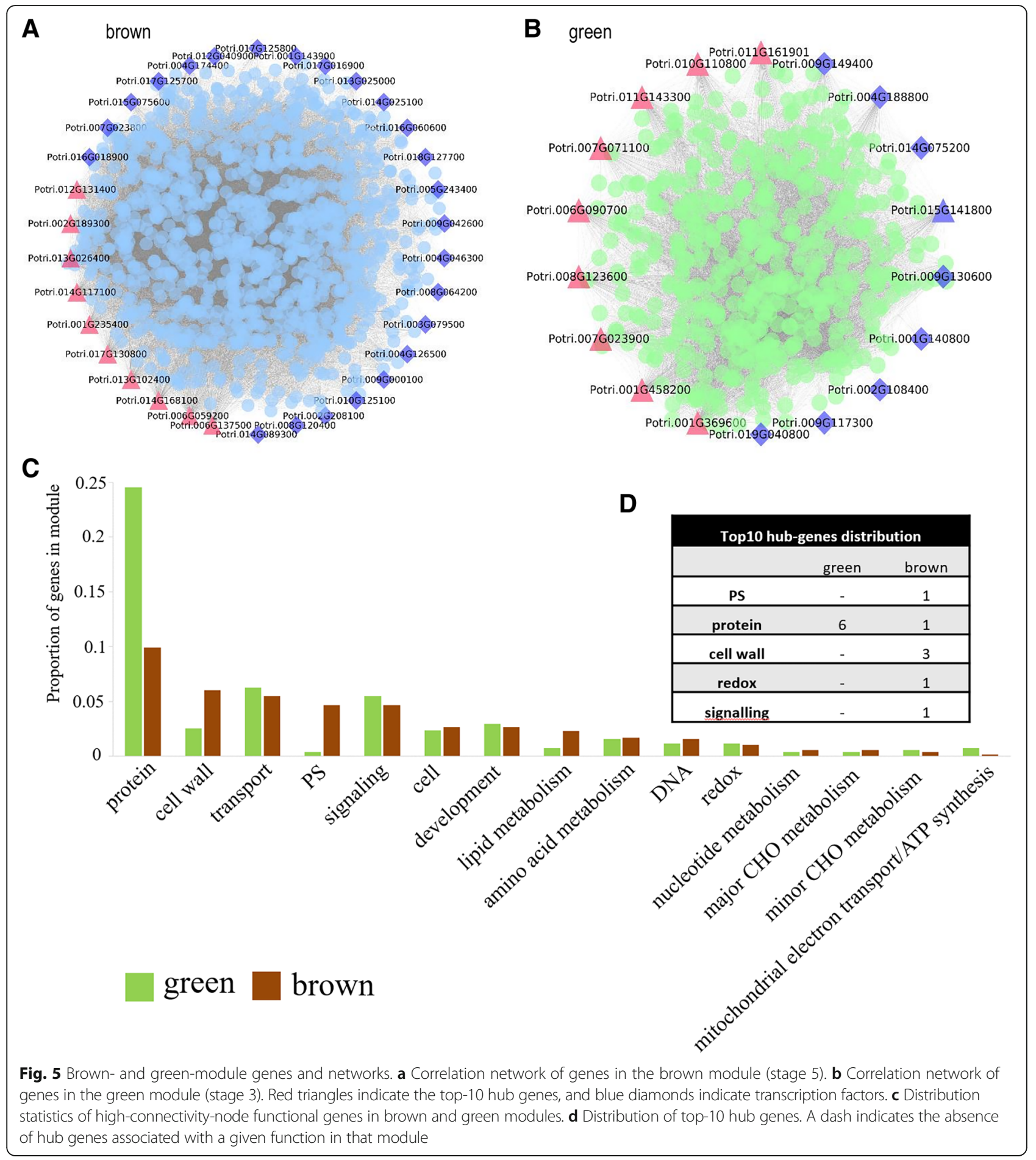

were identified. For example, phosphoenolpyruvate, an intermediate metabolite in the glycolytic pathway, is the product of enolase and is catalyzed by pyruvate kinase to yield pyruvate. Analysis of the correlation between the expression pattern of gene family members encoding the above-mentioned enzymes and the change trend of phosphoenolpyruvate revealed that potri.010 g254900 and potri.006 g116800 were highly correlated with phosphoenolpyruvate. Because pyruvate kinase, potri.010 g254900, is more closely related to phosphoenolpyruvate, this gene may play a key role in the pattern of phosphoenolpyruvate changes.

A strong positive correlation was uncovered between the change patterns of three metabolites: alpha-ketoglutarate, 


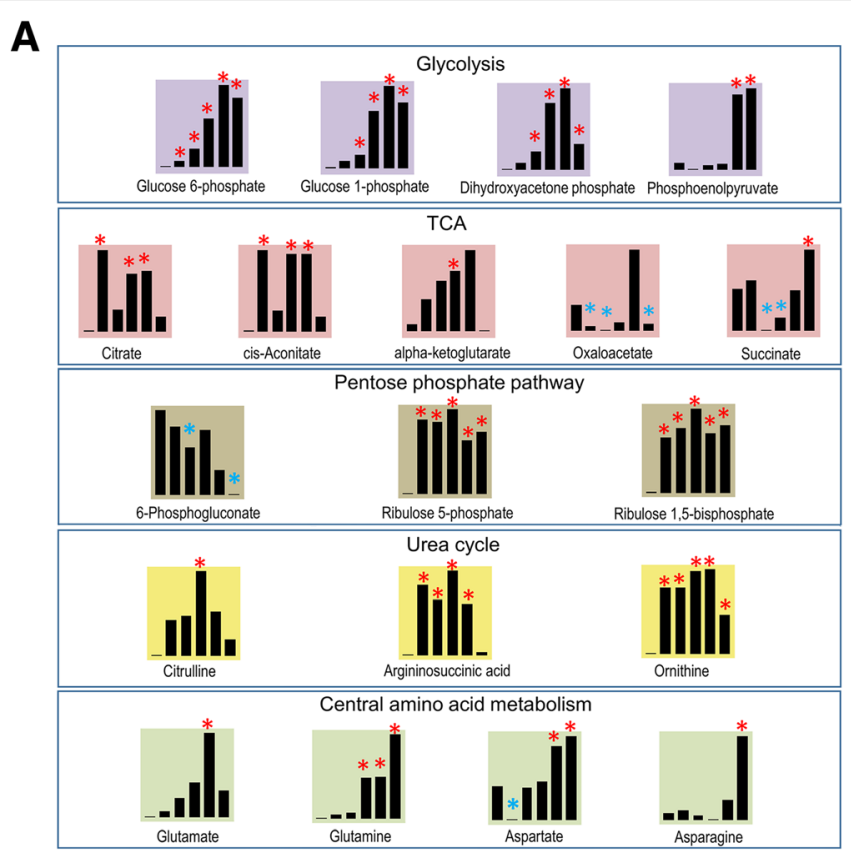

B

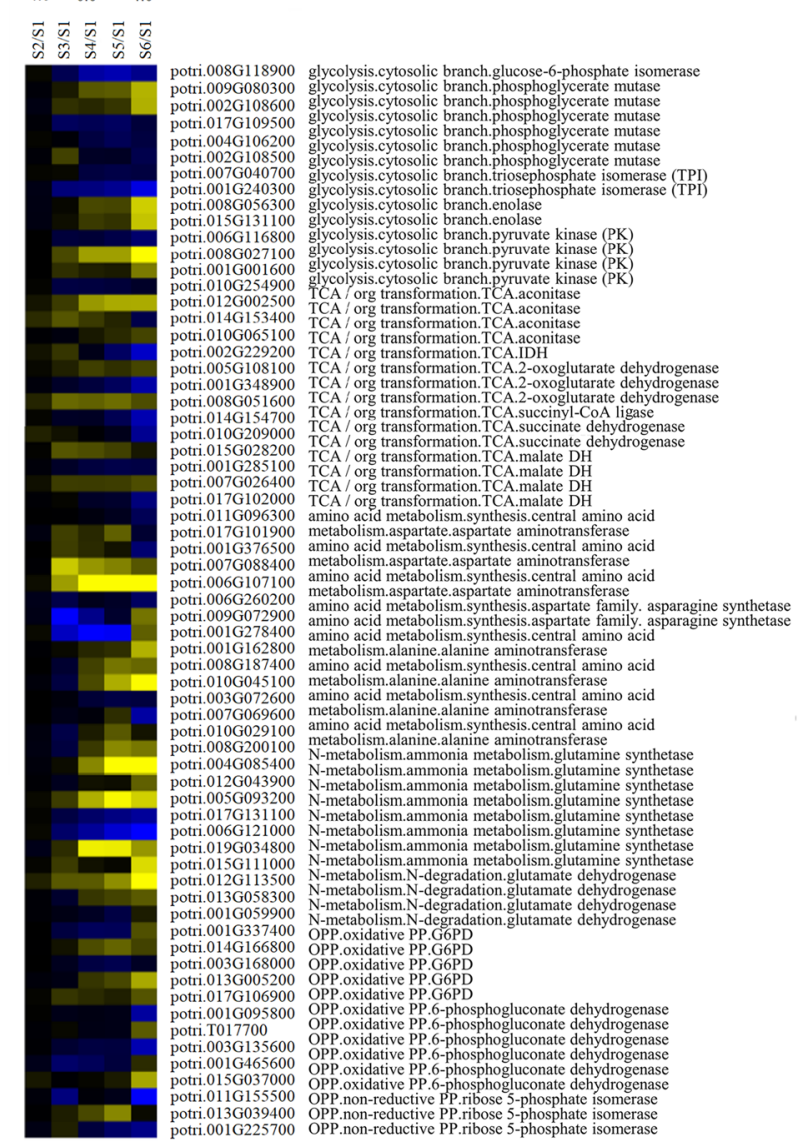

Fig. 6 (See legend on next page.) 
(See figure on previous page.)

Fig. 6 Profiling of primary metabolites and genes related to seed germination and post-germination processes. a Primary metabolite profiling of seed germination and post-germination processes. Asterisks indicate significant differences (red: significantly up-regulated; blue: significantly down-regulated). b Primary metabolism-related gene profiling of seed germination and post-germination processes. Expressions: blue, minimum; yellow, maximum. See also Additional file 8: Table S8 and Additional file 9: Table S9

glutamate, and glutamine. The two families of genes controlling the synthesis and catabolism of alpha-ketoglutarate were negatively correlated with all isocitrate dehydrogenase and alpha-ketoglutarate dehydrogenase genes except for potri.008 g051600 (encoding alpha-ketoglutarate dehydrogenation). In the glutamate synthesis pathway, genes encoding alanine aminotransferase and glutamate dehydrogenase generally were strongly positively correlated with alphaketoglutarate and glutamate. Genes encoding glutamine synthetase typically have features highly associated with glutamate and glutamine.

Adjacent metabolites in metabolic pathways generally have strongly related characteristics, and this correlation is typically positively correlated. As shown in Fig. 7, a positive correlation trend was found among most contiguous metabolites, which suggests that metabolite changes under the same pathway are generally consistent. In contrast, significant differences were found between glucose 6-phosphate and 6-phosphogluconate, 6-phosphogluconate and ribulose 5-phosphate, phosphoenolpyruvate and citric acid, and alpha-ketoglutarate and succinic acid, with a significant negative correlation observed between changes in the contents of these metabolites.

\section{Discussion}

Poplar is a model woody plant widely used in basic research. Because asexual reproduction is prevalent in poplar, the molecular mechanism of seed germination and its aftermath in poplar has not been investigated. In this study, samples from dry seed to seedling stages were collected and used for RNA-Seq and metabolomic analyses. Two different bioinformatic methods, $K$-means and WGCNA, were used to analyze the RNA-Seq data. This analysis produced several novel insights, including the identification of a series of time-specific modules, hub genes and transcription factors, some of the latter of which were consistent with previous research results. Many stress, cell wall, and protein-related genes were found to have a close relationship with germination and post-germination stages, while changes in glycolysis, oxidative phosphorylation, and the TCA were not significant. Further analysis showed that some metabolites in these pathways undergo significant changes at different stages of germination and that metabolite change patterns have similar characteristics under the same metabolic pathway. Enzymes associated with changes in metabolite content are commonly encoded by multiple gene families, and some members of these families were highly correlated with metabolite changes. Our study identified important genes and metabolites associated with germination and post-germination processes of poplar seeds and has laid a foundation for future biochemical and functional studies.

The seed germination process of Arabidopsis thaliana, rice, and soybean has been extensively studied. For example, two transcription factor genes, ZFP8 and WUSCHEL-related homeobox 11, as well as a $Y A B B Y$ family member are considered to be closely related to seed germination [25, 41, 42]. Howell et al. (2009) found that the transcriptional abundance of sugar metabolismrelated genes increased significantly in the late stage of rice seed germination [25]. Studies on the germination of barley seeds have shown that genes involved in amino acid metabolism are expressed at $48 \mathrm{~h}$ after imbibition [43]; this observation is consistent with the results shown in Fig. 4, which suggests that germination is a highly conserved process among higher plants. In contrast, however, we found that transcription factors such as $C P P(Z n)$ and $D O F$ are specifically expressed at different stages of seed germination, a phenomenon not observed in previous studies. We speculate that this discrepancy is due to differences among studied species.

Genes in green and brown modules in the WGCNA were found to be most relevant to stages 3 (slow water absorption period) and 5 (cotyledon expansion period), respectively. High-connectivity-node functional genes in green modules were associated with protein synthesis and decomposition, whereas those in brown modules were associated with $\mathrm{CHO}$, cell wall, lipid metabolism and PS. We speculate that changes first occur in the early protein metabolism pathway, with significant changes in saccharide metabolism taking place during the cotyledon expansion period. Because the WGCNA method does not allow discovery of gene modules most closely related to other periods, such as rapid water absorption or hypocotyl elongation, we were unable to detect events occurring at the transcriptional level at each stage of seed germination. We plan to explore this issue in future research.

In this study, we used two different bioinformatics algorithms, $K$-means and WGCNA, to analyze transcriptome data, a strategy similar to that applied in previous studies of strawberry and Brassica oleracea [44, 45]. Although these two methods provided us with different options for finding important biological events occurring 


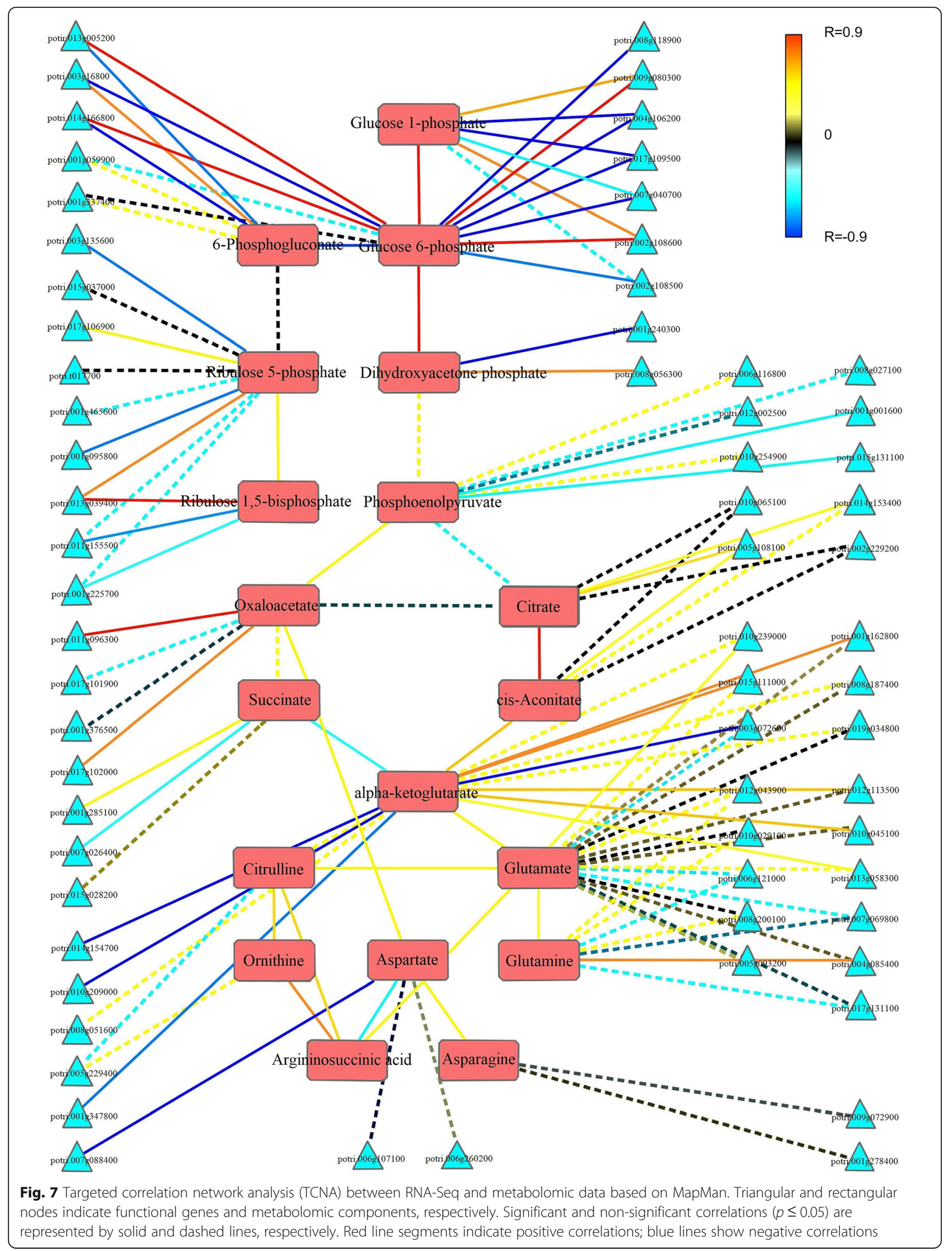


at different seed germination and post-germination stages, both suggested that glycolysis and the TCA cycle were in a steady state at the transcriptional level. This observation is inconsistent with previous findings [22, 25, 43, 46-51] and does not explain the changes in the metabolic levels of these two pathways. We thus needed to determine the causes of these metabolite changes and to identify the genes most likely closely related. Using the TCNA method, we were able to identify gene family members most closely related to changes in the content of specific metabolites. These results provide us with possible directions for subsequent determination of genes to be studied, and this combined method is also potentially useful for future multiomics joint analyses.

Correlation analysis of metabolites during the germination process revealed a significant positive correlation among the most relevant metabolites. A significant difference was found, however, between glucose 6-phosphate and 6-phosphogluconate, 6-phosphogluconate and ribulose 5-phosphate, phosphoenolpyruvate and citric acid, and alpha-ketoglutarate and succinic acid. These transformations are situated at junctions with other metabolic pathways, and a strong correlation exists between branch metabolites and upstream metabolites. We have therefore inferred that substances at these branch sites flow in multiple, major directions. These results can thus serve as a useful reference for further study of metabolite flow.

At the transcriptional level, many genes undergo complex changes during germination, even though the expression patterns of genes encoding the same enzyme are dissimilar. At the metabolic level, however, only a few significant changes to metabolites were observed (Fig. 6a, b). This result is probably due to the fact that a significant number of low-abundance metabolites cannot be detected by HPLC-MS/MS relative to RNA-Seq. Another possibility is that the level of transcription is a complex process: just a few genes may play a key role in a specific germination stage; in addition, post-transcriptional regulation may act as a buffer for this process, leading to very few changes at metabolic levels [52].

\section{Conclusions}

In this study, we investigated poplar seed germination and post-germination biological processes using a transcriptional combinatorial metabolomic method. In contrast to time-sequential methods used previously, we relied on a strategy based on morphological differences, which can better address the combination of molecular level and morphology. We compared our results with those obtained in earlier investigations of the germination of Arabidopsis [21, 53], rice [25], and barley [20,43] and found that seed germination is a highly conserved process among higher plants. Using a combination of $K$-means and WGCNA methods, we identified transcription factors and metabolic pathways playing an important role in specific stages of seed germination. The application of targeted network analysis methods allowed us to discover candidate genes participating in specific processes. Such a method also represents a possible strategy for future joint sequential multiomics analyses. Our study was the first to use an integrated approach in regard to seed germination in poplar. The results of our study are thus an important starting point for future analyses and provide new insights to explore alternative strategies and possibilities.

\section{Methods}

Plant materials, experimental conditions, and fresh weight measurements

Seeds of poplar (Poplar simonii $\times$ Poplar nigra) were collected from the same female poplar plants producing half-sib families at the Experimental Forest Farm of Northeast Forestry University (Harbin, China). Germination assays were carried out on three replicates (50 seeds per replicate). Seeds were incubated in covered plastic boxes at $25^{\circ} \mathrm{C}$ on two sheets of absorbent paper moistened with $4.5 \mathrm{~mL}$ of distilled water. After removing excess moisture with absorbent paper, 20 germinating seeds were weighed (to $\pm 0.0001 \mathrm{~g}$ ) every $15 \mathrm{~min}$ at an ambient temperature of $25 \pm 1{ }^{\circ} \mathrm{C}$. Transcriptomic and metabolic profiling was performed at specific time points based on the fresh weights of germinated seeds. After weighing, seeds were immediately blotted with absorbent paper, snap frozen in liquid nitrogen, and subjected to transcriptomic and metabolic analyses.

\section{Transcriptome profiling and analysis}

Total RNA was extracted from approximately 200 seeds using Trizol reagent (Invitrogen, Carlsbad, CA, USA) and then incubated with $10 \mathrm{U}$ DNase I (Takara, Dalian, China) for $30 \mathrm{~min}$ at $37^{\circ} \mathrm{C}$ to remove genomic DNA. Three experimental replicates per sample were used for RNA library construction. RNA-Seq libraries were prepared according to the Illumina Library Prep Kit manufacturer's protocol. After sequencing, raw data were filtered to remove adaptor contamination and low-quality reads. All clean reads were then mapped to the poplar genome, which was downloaded from the Phytozome website (https://phytozome.jgi.doe.gov/pz/portal.html), using the HISAT2 alignment algorithm with default parameters [54]. Mapped reads of each gene were extracted using SAMtools [55]. DEGs were identified in NOISeq using fold change $\geq 2$ and $p \geq 0.8$ thresholds [56]. Gene expression levels were normalized using the FPKM method.

\section{Clustering of gene expression data}

$K$-means clustering with Euclidean distances in MeV4.8 [57] yielded 24 clusters based on input FPKM values. Coexpression networks were constructed using the 
WGCNA package in $\mathrm{R}$ [58]. Eigengene values were calculated for each module and used to test associations with each germination stage. Subsequent analyses were performed using $r \geq 0.75$ and $p \leq 0.05$ thresholds (Student's $t$-test). Networks were visualized using Cytoscape v.3.5.1 [59].

\section{Enrichment analysis}

The MapMan tool was used to assign pathway categories and screen transcription factors [37]. Fisher's exact test was used to identify categories significantly enriched in DEGs ( $p \leq 0.05$ threshold).

\section{Metabolomic analysis}

Analysis of metabolites was performed using $50 \mathrm{mg}$ of poplar seed collected from different germination periods, LC-MS/MS analyses were carried out on an UHPLC system (1290, Agilent Technologies, USA) equipped with a UPLC BEH Amide column $(1.7 \mu \mathrm{m}, 2.1 \times 100$ $\mathrm{mm}$, Waters) coupled to a TripleTOF 6600 system (QTOF, AB Sciex) operating in EI mode. The mobile phase (A) consisted of $25 \mathrm{mM} \mathrm{NH}_{4} \mathrm{OAc}$ and $25 \mathrm{mM}$ aqueous $\mathrm{NH}_{4} \mathrm{OH}(\mathrm{pH}=9.75)$. Gradient elution was carried out at a delivery rate of $0.3 \mathrm{~mL} \mathrm{~min}^{-1}$ using the mobile phase (A) and acetonitrile (B) as follows: $0 \mathrm{~min}, 85 \% \mathrm{~B} ; 2 \mathrm{~min}$, $75 \% \mathrm{~B} ; 9 \mathrm{~min}, 0 \% \mathrm{~B} ; 14 \mathrm{~min}, 0 \% \mathrm{~B} ; 15 \mathrm{~min}, 85 \% \mathrm{~B}$; and $20 \mathrm{~min}, 85 \% \mathrm{~B}$. The injection volume was $2 \mu \mathrm{L}$. Six biological repeats were performed, with all other details as described in a previous study [60]. During data processing, two steps were used to determine significant differences in metabolites at different times. In the first step, first principal component of variable importance in the projection (VIP) values were obtained. Changed metabolites were considered to be those with VIP values exceeding 1.0. In the second step, the remaining variables were assessed by Student's $t$-test $(p>0.05)$, and variables were discarded between the two comparison groups [61].

\section{TCNA}

TCNA is used to evaluate the contribution of each multigene family member to the change in a target metabolite. In this method, gene family members encoding proteins directly involved in the "synthesis" and "decomposition" of a target metabolite are considered. Genes responsible for target metabolite synthesis and decomposition were identified on the basis of gene annotation and metabolite rankings obtained from annotation information generated with the MapMan tool. A Spearman correlation analysis was performed and visualization was carried out using Cytoscape software (3.5.1) based on $p \geq 0.05$ (Student's $t$-test) and $\mathrm{r} \geq 0.25$ thresholds.

\section{Additional files}

Additional file 1: Table S1. Changes in fresh weight of poplar seeds

Additional file 2: Table S2. The average number of raw reads per library and matching results. (XLSX $17 \mathrm{~kb}$ )

Additional file 3: Table S3. IDs of genes in the 24 clusters. (XLSX $162 \mathrm{~kb}$ )

Additional file 4: Table S4. The relative abundance of transcription factors at different stages. (XLSX $17 \mathrm{~kb}$ )

Additional file 5: Table S5. Enriched pathway categories and mobilization trends in each module. (XLSX $22 \mathrm{~kb}$ )

Additional file 6: Table S6. High-connectivity transcription factors. (XLSX 16 kb)

Additional file 7: Table S7. Brown module and green module functional gene IDs and connectivity. (XLSX 48 kb)

Additional file 8: Table S8. Primary metabolite changes during seed germination and post germination. (XLSX $10 \mathrm{~kb}$ )

Additional file 9: Table S9. Primary metabolism-related gene profiling. (XLSX 22 kb)

Additional file 10: Table S10. Genes and correlations involved in a targeted correlation network analysis (TCNA). (XLSX 14 kb)

\section{Abbreviations}

AP2/EREBP: APETALA2/ethylene-responsive element binding proteins; CPP $(Z n)$ : Cystein rich polycomb like protein (Zn); DEGs: Differentially expressed genes; DOF: DNA binding with one finger; EIL: Ethylene-insensitive3 -like; HPLC-MS/MS: High-performance liquid chromatography-mass spectrometry/ mass spectrometry; ME: Module eigengene; TCA: Tricarboxylic acid cycle; TCNA: Targeted correlation network analysis; VIP: Variable importance in the projection; WGCNA: Weighted gene coexpression network analysis

\section{Acknowledgements}

Not applicable.

\section{Authors' contributions}

CQ analyzed the RNA-Seq and metabolomic data. CQ, LC, and LL wrote and revised the manuscript. $Z Z$ performed the RNA experiment and statistical analysis. $C Q, J H, X S, P Z, C Y, Z X$, and $G L$ designed the study and organized this work. All authors contributed to and approved the final manuscript.

\section{Funding}

This study was supported by the Fundamental Research Funds for the Central Universities (2572017CA01), National Natural Science Foundation of China (No. 31570648), and National Natural Science Foundation of China (No. 31600534). The funding agencies were not involved in study design, data collection and analysis, or preparation of the manuscript.

\section{Availability of data and materials}

All datasets generated or analyzed during this study are available from the corresponding author upon reasonable request.

Ethics approval and consent to participate Not applicable.

\section{Consent for publication}

Not applicable.

\section{Competing interests}

The authors declare that they have no competing interest.

\section{Author details}

${ }^{1}$ State Key Laboratory of Tree Genetics and Breeding (Northeast Forestry University), School of Forestry, Northeast Forestry University, Harbin 150040, People's Republic of China. ${ }^{2}$ School of Forestry, Northeast Forestry University, Harbin 150040, People's Republic of China. ${ }^{3}$ College of Life Science, 
Northeast Forestry University, Harbin 150040, People's Republic of China. ${ }^{4}$ Key Laboratory of Saline-Alkali Vegetation Ecology Restoration, Ministry of Education, Alkali Soil Natural Environmental Science Center, Northeast Forestry University, Harbin 150040, People's Republic of China.

\section{Received: 15 January 2019 Accepted: 31 May 2019} Published online: 26 June 2019

\section{References}

1. Bewley JD. Seed germination and dormancy. Plant Cell. 1997;9(7):1055-66.

2. Bewley JD, Bradford KJ, Hilborst HWM, Nonogaki H. Seeds - Physiology of Development, Germination and Dormancy. 3rd ed. New York: Springer; 2013.

3. Koornneef $M$, Bentsink $L$, Hilhorst $H$. Seed dormancy and germination. Curr Opin Plant Biol. 2002;5(1):33-6.

4. Miransari M, Smith DL. Plant hormones and seed germination. Environ Exp Bot. 2014;99:110-21.

5. Rajjou L, Duval M, Gallardo K, Catusse J, Bally J, Job C, Job D. Seed germination and vigor. Annu Rev Plant Biol. 2012;63:507-33.

6. Macquet A, Ralet MC, Kronenberger J, Marion-Poll A, North HM. In situ, chemical and macromolecular study of the composition of Arabidopsis thaliana seed coat mucilage. Plant \& cell physiology. 2007;48(7):984-99.

7. Robert C, Noriega A, Tocino A, Cervantes E. Morphological analysis of seed shape in Arabidopsis thaliana reveals altered polarity in mutants of the ethylene signaling pathway. J Plant Physiol. 2008;165(9):911-9.

8. Windsor JB, Symonds W, Mendenhall J, Lloyd AM. Arabidopsis seed coat development: morphological differentiation of the outer integument. Plant J. 2000;22(6):483-93.

9. Yamaguchi S, Kamiya Y, Sun T. Distinct cell-specific expression patterns of early and late gibberellin biosynthetic genes during Arabidopsis seed germination. Plant J. 2001;28(4):443-53.

10. Datta K, Parker H, Averyhart-Fullard V, Schmidt A, Marcus A. Gene expression in the soybean seed axis during germination and early seedling growth. Planta. 1987;170(2):209-16.

11. Dierking EC, Bilyeu KD. Raffinose and stachyose metabolism are not required for efficient soybean seed germination. J Plant Physiol. 2009; 166(12):1329-35

12. Wang C, Wang H, Zhang J, Chen S. A seed-specific AP2-domain transcription factor from soybean plays a certain role in regulation of seed germination. Sci China C Life Sci. 2008:51(4):336-45.

13. Asakura T, Watanabe $H$, Abe K, Arai S. Rice aspartic proteinase, oryzasin, expressed during seed ripening and germination, has a gene organization distinct from those of animal and microbial aspartic proteinases. Eur J Biochem. 1995;232(1):77-83.

14. Assmann SM. G protein signaling in the regulation of rice seed germination. Sci STKE. 2005;2005(310):cm12.

15. Mitsunaga S, Kawakami O, Numata T, Yamaguchi J, Fukui K, Mitsui T. Polymorphism in rice amylases at an early stage of seed germination. Biosci Biotechnol Biochem. 2001;65(3):662-5.

16. Palmiano EP, Juliano BO. Changes in the activity of some hydrolases, peroxidase, and catalase in the Rice seed during germination. Plant Physiol. 1973;52(3):274-7.

17. Taylor NL, Howell KA, Heazlewood JL, Tan TYW, Narsai R, Huang SB, Whelan J, Millar AH. Analysis of the Rice mitochondrial carrier family reveals anaerobic accumulation of a basic amino acid carrier involved in arginine metabolism during seed germination. Plant Physiol. 2010;154(2):691-704.

18. Abdul-Baki AA. Metabolism of barley seed during early hours of germination. Plant Physiol. 1969;44(5):733-8.

19. Martinez M, Cambra I, Carrillo L, Diaz-Mendoza M, Diaz I. Characterization of the entire cystatin gene family in barley and their target cathepsin L-like cysteine-proteases, partners in the hordein mobilization during seed germination. Plant Physiol. 2009;151(3):1531-45.

20. Potokina E, Sreenivasulu N, Altschmied L, Michalek W, Graner A. Differentia gene expression during seed germination in barley (Hordeum vulgare L.). Funct Integr Genomics. 2002;2(1-2):28-39.

21. Dekkers BJW, Pearce $S$, van Bolderen-Veldkamp RP, Marshall A, Widera P, Gilbert J, Drost HG, Bassel GW, Muller K, King JR, et al. Transcriptional dynamics of two seed compartments with opposing roles in Arabidopsis seed germination. Plant Physiol. 2013;163(1):205-15.

22. Fait A, Angelovici R, Less H, Ohad I, Urbanczyk-Wochniak E, Fernie AR, Galili G. Arabidopsis seed development and germination is associated with temporally distinct metabolic switches. Plant Physiol. 2006;142(3):839-54.
23. Gallardo K, Job C, Groot SP, Puype M, Demol H, Vandekerckhove J, Job D. Proteomic analysis of arabidopsis seed germination and priming. Plant Physiol. 2001;126(2):835-48.

24. Girke T, Todd J, Ruuska S, White J, Benning C, Ohlrogge J. Microarray analysis of developing Arabidopsis seeds. Plant Physiol. 2000;124(4):1570-81.

25. Howell KA, Narsai R, Carroll A, Ivanova A, Lohse M, Usadel B, Millar AH, Whelan J. Mapping metabolic and transcript temporal switches during germination in rice highlights specific transcription factors and the role of RNA instability in the germination process. Plant Physiol. 2009;149(2):961-80.

26. Wang WQ, Liu SJ, Song SQ, Moller IM. Proteomics of seed development, desiccation tolerance, germination and vigor. Plant Physiol Biochem. 2015;86:1-15.

27. Yang $\mathrm{P}$, Li X, Wang $X$, Chen $H$, Chen F, Shen S. Proteomic analysis of rice (Oryza sativa) seeds during germination. Proteomics. 2007;7(18):3358-68.

28. Zhang H, Wang WQ, Liu SJ, Moller IM, Song SQ. Proteome analysis of poplar seed vigor. PLoS One. 2015;10(7):e0132509.

29. Marquis DA. Seed storage and germination under northern hardwood forests. Can J For Res. 1975;5(3):478-84.

30. Bajaj YPS. Biotechnology of tree improvement for rapid propagation and biomass energy production. Trees I. Berlin: Springer; 1986. p. 1-23.

31. Karnosky DF. Potential for forest tree improvement via tissue culture. BioScience. 1981;31(2):114-20.

32. Dewan S, Vander Mijnsbrugge K, De Frenne P, Steenackers M, Michiels B, Verheyen K. Maternal temperature during seed maturation affects seed germination and timing of bud set in seedlings of European black poplar. For Ecol Manag. 2018;410:126-35.

33. Madejon P, Cantos M, Jimenez-Ramos MC, Maranon T, Murillo JM. Effects of soil contamination by trace elements on white poplar progeny: seed germination and seedling vigour. Environ Monit Assess. 2015;187(11):663.

34. Zhang H, Zhou KX, Wang WQ, Liu SJ, Song SQ. Proteome analysis reveals an energy-dependent central process for poplar seed germination. J Plant Physiol. 2017;213:134-47.

35. Finch-Savage WE, Leubner-Metzger G. Seed dormancy and the control of germination. New Phytol. 2006;171(3):501-23.

36. Weitbrecht K, Muller K, Leubner-Metzger G. First off the mark: early seed germination. J Exp Bot. 2011;62(10):3289-309.

37. Thimm $O$, Bläsing $O$, Gibon $Y$, Nagel A, Meyer S, Krüger $P$, Selbig J, Müller LA, Rhee SY, Stitt M. Mapman: a user-driven tool to display genomics data sets onto diagrams of metabolic pathways and other biological processes. Plant J. 2004;37(6):914-39.

38. Kubo H, Hayashi K. Characterization of root cells of anl2 mutant in Arabidopsis thaliana. Plant Sci. 2011;180(5):679-85.

39. Kubo H, Peeters AJ, Aarts MG, Pereira A, Koornneef M. ANTHOCYANINLESS2, a homeobox gene affecting anthocyanin distribution and root development in Arabidopsis. Plant Cell. 1999;11(7):1217-26.

40. Dobrowolska I, Businge E, Abreu IN, Moritz T, Egertsdotter U. Metabolome and transcriptome profiling reveal new insights into somatic embryo germination in Norway spruce (Picea abies). Tree Physiol. 2017;37(12):1752-66.

41. Kristof JR, Coppersmith JL, Hong K, Liu PP, Homrichhausen TM, Sun J, Martin RC, Nonogaki H. An Arabidopsis thaliana embryo arrest mutant exhibiting germination potential. Seed Sci Res. 2008;18(2):55-65.

42. Shamimuzzaman M, Vodkin L. Genome-wide identification of binding sites for NAC and YABBY transcription factors and co-regulated genes during soybean seedling development by ChIP-Seq and RNA-Seq. BMC Genomics. 2013:14:477.

43. Sreenivasulu N, Usadel B, Winter A, Radchuk V, Scholz U, Stein N, Weschke W, Strickert $M$, Close TJ, Stitt M. Barley grain maturation and germination: metabolic pathway and regulatory network commonalities and differences highlighted by new MapMan/PageMan profiling tools. Plant Physiol. 2008;146(4):1738-58.

44. Hollender CA, Kang C, Darwish O, Geretz A, Matthews BF, Slovin J, Alkharouf $\mathrm{N}$, Liu Z. Floral transcriptomes in woodland strawberry uncover developing receptacle and anther gene networks. Plant Physiol. 2014;165(3):1062-75.

45. Wu S, Lei J, Chen G, Chen H, Cao B, Chen C. De novo transcriptome assembly of Chinese kale and global expression analysis of genes involved in Glucosinolate metabolism in multiple tissues. Front Plant Sci. 2017:8:92.

46. Angelovici R, Fait A, Fernie AR, Galili G. A seed high-lysine trait is negatively associated with the TCA cycle and slows down Arabidopsis seed germination. New phytol. 2011;189(1):148-59.

47. Eastmond PJ, Graham IA. Re-examining the role of the glyoxylate cycle in oilseeds. Trends Plant Sci. 2001;6(2):72-8. 
48. He D, Han C, Yao J, Shen S, Yang P. Constructing the metabolic and regulatory pathways in germinating rice seeds through proteomic approach. Proteomics. 2011;11(13):2693-713.

49. Kelly AA, Quettier AL, Shaw E, Eastmond PJ. Seed storage oil mobilization is important but not essential for germination or seedling establishment in Arabidopsis. Plant Physiol. 2011;157(2):866-75.

50. Rosental L, Nonogaki H, Fait A. Activation and regulation of primary metabolism during seed germination. Seed Sci Res. 2014:24(01):1-15.

51. Soeda Y, Konings MC, Vorst O, van Houwelingen AM, Stoopen GM, Maliepaard CA, Kodde J, Bino RJ, Groot SP, van der Geest AH. Gene expression programs during Brassica oleracea seed maturation, osmopriming, and germination are indicators of progression of the germination process and the stress tolerance level. Plant Physiol. 2005;137(1):354-68.

52. Amiour N, Imbaud S, Clement G, Agier N, Zivy M, Valot B, Balliau T, Armengaud P, Quillere I, Canas R, et al. The use of metabolomics integrated with transcriptomic and proteomic studies for identifying key steps involved in the control of nitrogen metabolism in crops such as maize. J Exp Bot. 2012;63(14):5017-33.

53. Kimura M, Nambara E. Stored and neosynthesized mRNA in Arabidopsis seeds: effects of cycloheximide and controlled deterioration treatment on the resumption of transcription during imbibition. Plant Mol Biol. 2010;73(1):119-29.

54. Kim D, Langmead B, Salzberg SL. HISAT: a fast spliced aligner with low memory requirements. Nat Methods. 2015;12(4):357-60.

55. Li H, Handsaker B, Wysoker A, Fennell T, Ruan J, Homer N, Marth G, Abecasis G, Durbin R. Genome project data processing S: the sequence alignment/ map format and SAMtools. Bioinformatics. 2009;25(16):2078-9.

56. Tarazona S, Furio-Tari P, Turra D, Pietro AD, Nueda MJ, Ferrer A, Conesa A. Data quality aware analysis of differential expression in RNA-seq with NOISeq R/bioc package. Nucleic Acids Res. 2015;43(21):e140.

57. Saeed Al, Sharov V, White J, Li J, Liang W, Bhagabati N, Braisted J, Klapa M, Currier T, Thiagarajan M, et al. TM4: a free, open-source system for microarray data management and analysis. Biotechniques. 2003;34(2):374-8.

58. Langfelder P, Horvath S. WGCNA: an R package for weighted correlation network analysis. BMC bioinformatics. 2008;9:559.

59. Shannon P, Markiel A, Ozier O, Baliga NS, Wang JT, Ramage D, Amin N, Schwikowski B, Ideker T. Cytoscape: a software environment for integrated models of biomolecular interaction networks. Genome Res. 2003;13(11): 2498-504.

60. Want EJ, Wilson ID, Gika H, Theodoridis G, Plumb RS, Shockcor J, Holmes E, Nicholson JK. Global metabolic profiling procedures for urine using UPLCMS. Nat Protoc. 2010;5(6):1005-18.

61. Smith CA, Want EJ, O'Maille G, Abagyan R, Siuzdak G. XCMS: processing mass spectrometry data for metabolite profiling using nonlinear peak alignment, matching, and identification. Anal Chem. 2006;78(3):779-87.

\section{Publisher's Note}

Springer Nature remains neutral with regard to jurisdictional claims in published maps and institutional affiliations.

Ready to submit your research? Choose BMC and benefit from:

- fast, convenient online submission

- thorough peer review by experienced researchers in your field

- rapid publication on acceptance

- support for research data, including large and complex data types

- gold Open Access which fosters wider collaboration and increased citations

- maximum visibility for your research: over $100 \mathrm{M}$ website views per year

At BMC, research is always in progress.

Learn more biomedcentral.com/submissions 